\title{
Hysteretic Bifurcation Model of the Boost Converter
}

\author{
Denis PELIN, Željko STOJANOVIĆ
}

\begin{abstract}
Steady-state responses of the boost converter by change of input voltage are experimentally identified. In a part of parameter space hysteretic behaviour of steadystate responses by varying input voltage in different tendency of change is noticed. Procedure for studying steady-state responses of the boost converter is proposed by using one-parameter and two-parameter bifurcation diagrams as simulation result. It is shown that the initial condition of bifurcation parameters affects steady-state responses inside hysteretic region. There is a variety of combinations of initial inductor current and initial capacitor voltage which cause particular steady-state. Therefore, hysteretic bifurcation model of the boost converter is proposed and verified by simulations and measurement outside hysteretic region.
\end{abstract}

Keywords: bifurcations; boost converter; hysteretic behaviour; simulation; steady-state; two-parameter bifurcation diagram

\section{INTRODUCTION}

The boost converters are a widespread type of DC/DC power electronics converters which are used in many applications such as power factor correction circuits, MPPT for photovoltaic systems, hand held applications, automotive power supplies and other [1-4]. DC/DC converters are time-varying nonlinear dynamical systems, so they manifest various nonlinear phenomena like different types of bifurcations, periodicities and chaos [58]. Study of these phenomena is necessary in order to design reliable converters. Although boost converters are intended for functioning in the way that period of state variables is equal to the intended switching period, they are prone to change the period of operation. Those changes of the period of operation so called bifurcations can cause unwanted phenomena like increased ripple of state variables and audible noises which can lead to components damage or shortening of lifetime [5, 8, 9].

One of numerous phenomena characterizing nonlinear systems is hysteretic behaviour which is obtained by change of bifurcation parameters. Hysteretic behaviour is a well-known phenomenon in many technical systems like magnetic hysteresis, Schmitt trigger hysteresis, thermostat hysteresis, memristor hysteresis, etc. Hysteretic behaviour of steady-states means that change of corresponding bifurcation parameter in one tendency and then back in opposite tendency to starting value will cause change from one type of steady-state response to another type of steadystate response and vice versa at different value of bifurcation parameter $[10,11]$. It is caused by memory effect. Memory effect is often a consequence of components having energy storing properties like capacitors and inductors which are building components of every boost converter. Hysteretic behaviour is always associated by coexistence of two attractors at the same parameter value $[10,11]$. For a successful application of the boost converter it is essential to identify hysteretic region regarding converter parameters as well as the variation of input or output variables. In this way it is possible to avoid the possibility of unintended skipping between coexisting steady-states due to noise, ripple or other disturbances $[7,9,12,14]$.

Coexisting attractors related to hysteretic behaviour in various $\mathrm{DC} / \mathrm{DC}$ converters were studied in few papers.
Coexisting attractors were considered by simulation in [1315]. In work [16] it was predicted by simulation of nonlinear system and experimentally confirmed in the buck converter. Regarding the type of transition between steadystate responses coexisting attractors of periodic and quasi periodic steady-states were studied in $[13,14,16]$ and coexisting attractors of two different periodic steady-states were studied in $[12,15]$. Commonly used tool for identification of hysteretic behaviour is one-parameter bifurcation diagram. It is sufficient to show the values of bifurcation parameter in range of hysteretic region. Twoparameter bifurcation diagrams are proposed in [15] but they are much more time consuming than one-parameter bifurcation diagram.

As mentioned before, memory effect causing hysteretic behaviour is correlated by sensitivity of initial conditions of the state variables. Impact of initial conditions on appearance of coexisting attractors was considered in [13-15]. In order to get a deeper insight in hysteretic behaviour mechanism the two-parameter bifurcation diagrams are particularly convenient. Unlike in one parameter bifurcation diagram, in two-parameter bifurcation diagram much clearer overview of the zones of operation is obtained and the correct choice of converter parameters is facilitated. Researchers should keep in mind that steady-state responses in vicinity of hysteretic region are extremely sensitive on circuit parameters and initial conditions. Moreover, "small" variations of circuit parameters and initial conditions are not under control of researcher as is considered in [17].

In this paper a hysteretic behaviour of steady-state responses regarding the tendency of change of input voltage is obtained both experimentally and by simulation. By increasing input voltage the converter exhibits sequence from period-1 steady-state through period-2 steady-state to period- 6 steady-state and by decreasing input voltage in the same range converter exhibits sequence from period-6 steady-state to period-3 steady-state. Observed hysteretic behaviour exhibits transitions between different periodic steady-states for each tendency of change of bifurcation parameter. Identified behaviour is differed from behaviours which had been reported in previous studies in which hysteretic behaviour exhibited transitions between the same periodic steady-state responses. The objective of the paper is to research mutual 
dependence of circuit parameters and initial conditions on appearance of hysteretic behaviour. In order to obtain hysteretic bifurcation model of the boost converter, twoparameter bifurcation diagrams are proposed with initial conditions of state variables as bifurcation parameters. It is shown that proper combination of initial conditions leads to the hysteretic behaviour of the converter.

\section{IDENTIFICATION OF HYSTERETIC BEHAVIOR BY MEASUREMENT}

The boost converter is realised with PWM using an integrated circuit MC34060A, Fig. 1. Fully controllable switch, (transistor BD901) is driven by the integrated circuit. When the proportional part of output voltage is higher than integrated circuit's internal saw tooth ramp voltage $u_{\text {ramp }}$ the controllable switch is in the OFF-state and otherwise is in the $\mathrm{ON}$-state. Uncontrollable switch is realised by diode BYW95C. The state of uncontrollable switch depends on the state of controllable switch and inductor current.

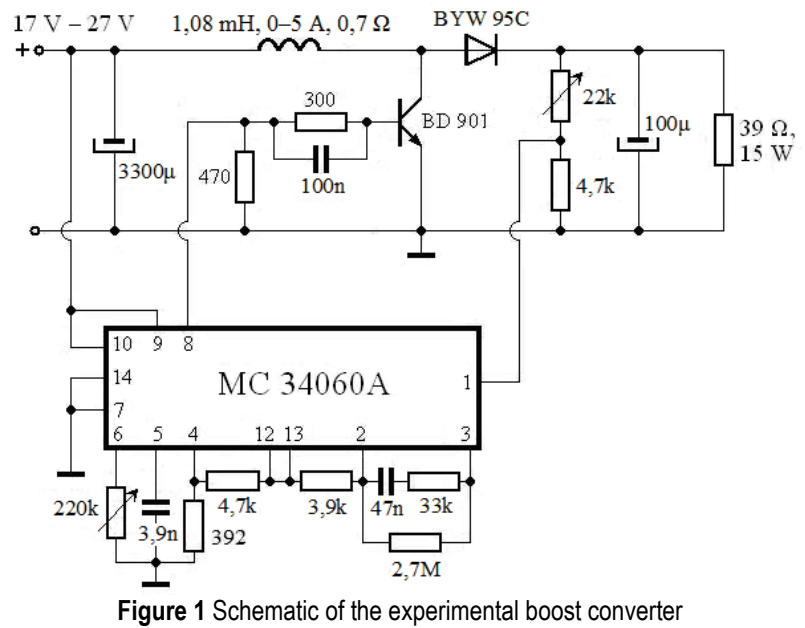

Thus, the switching intervals are dictated by saw tooth voltage period $T=200 \mu$ s. The saw tooth voltage period is small enough to ensure that high frequency effects could be neglected. The value of saw tooth voltage period was adjusted with variable resistor connected to the pin 6 of the MC34060A. The average value of output voltage was set from $27 \mathrm{~V}$ to $28 \mathrm{~V}$. It was adjusted by changing the feedback using variable resistor connected to the pin 1 of the MC34060A. The input voltage was set from $17 \mathrm{~V}$ to $27 \mathrm{~V}$ in order to study steady-state responses of the converter.

\subsection{Bifurcation Behaviour by Change of Input Voltage}

Measurements are obtained by taking into consideration waveforms of state variables although any of common measurement methods for obtained period can be used. It is sufficient to show waveforms of just one state variable to identify the type of steady-state response. The transition from one circuit topology to another and identification of steady-state response is more distinguishable by observing of inductor current than capacitor voltage. So, inductor current was chosen.

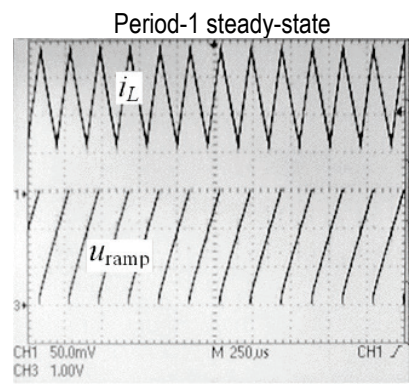

a)

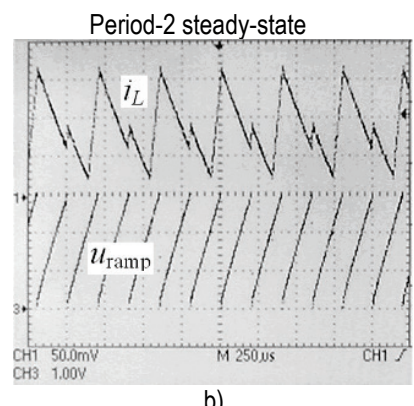

b)
Figure 2 Steady-states obtained by increasing of input voltage. (a) Input voltage $E=17,81 \mathrm{~V}$; (b) Input voltage $E=22,89 \mathrm{~V}$. Sensitivity $0,5 \mathrm{~A} /$ div, $1 \mathrm{~V} /$ div, $250 \mu \mathrm{s} /$ div.

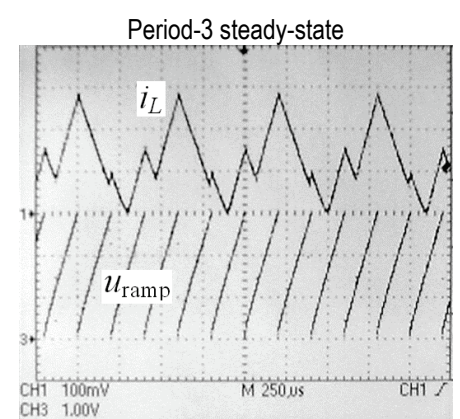

Figure 3 Steady-state obtained by decreasing of input voltage. Input voltage $E=22,03 \mathrm{~V}$. Sensitivity $1 \mathrm{~A} / \mathrm{div}, 1 \mathrm{~V} / \mathrm{div}, 250 \mu \mathrm{s} / \mathrm{div}$.

There are two series of results regarding the tendency of changing input voltage. By increasing input voltage the converter exhibits period-1 steady-state, period-2 steadystate and periodic steady-states of higher period interchanging with chaotic steady-states. Inductor current waveforms of period-1 steady-state and period-2 steadystate are shown together with associated sawtooth voltage in Fig. 2. The type of steady-state is identified by comparison of inductor current period and sawtooth voltage period. In period- $N$ steady-state the period of inductor current is $N$ times longer than the sawtooth voltage period. In chaotic steady-state the period of inductor current is infinite. By decreasing input voltage the converter exhibits the same sequence of steady-states in opposite order as by increasing input voltage. At the end of sequence the converter exhibits period-3 steady-state, Fig. 3, as opposed to period-2 steady-state and period-1 steady-state. Steady-states of the converter obtained for both tendencies of input voltage are shown in chart in Fig. 4. Each steady-state is marked by different colour.

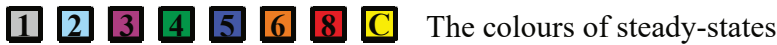

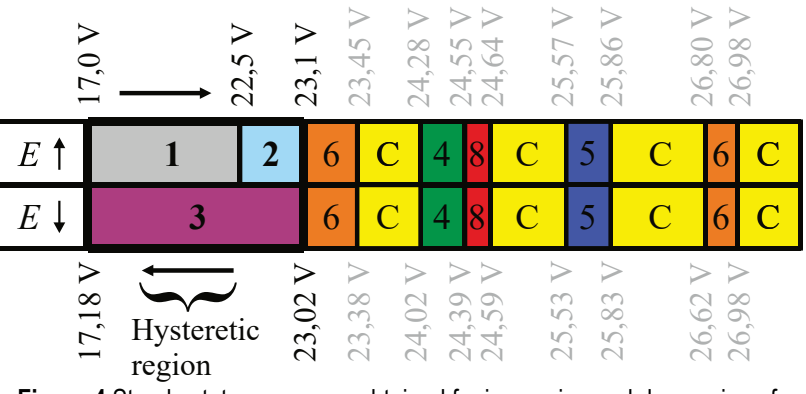

Figure 4 Steady-state responses obtained for increasing and decreasing of input voltage $E$ and qualitative comparison of converters steady-states. 


\subsection{Hysteretic Region}

In both series of results there is the same sequence of steady-states in range from $23,1 \mathrm{~V}$ to $27 \mathrm{~V}$, while series differ in range from $17,18 \mathrm{~V}$ to $23,1 \mathrm{~V}$.

In the region of difference by increasing the input voltage the converter exhibits period-1steady-state and period-2 steady-state, while by decreasing the input voltage the converter exhibits period-3 steady-state. This region of coexistence of two different steady-state solutions is called hysteretic region. Presented type of hysteretic behaviour was not found in available literature.

It is necessary to find a procedure for obtaining hysteretic bifurcation model of boost converter in order to find specific values of input voltage as well as the initial conditions of steady-state variables which characterise hysteretic region.

\section{PROPOSED PROCEDURE FOR MODELING HYSTERETIC BEHAVIOUR \\ 3.1 Mathematical Model of the Converter}

The equivalent circuit of boost converter is shown in Fig. 5. Both modes of operation, continuous conduction mode and discontinuous conduction mode are assumed. Therefore, there are three possible power circuit topologies in a period of operation $T=1 / f$. The boost converter can be thought of as a piece-wise linear time-varying network. Thus, each of the possible circuit topologies is modelled by a different set of linear differential equations.

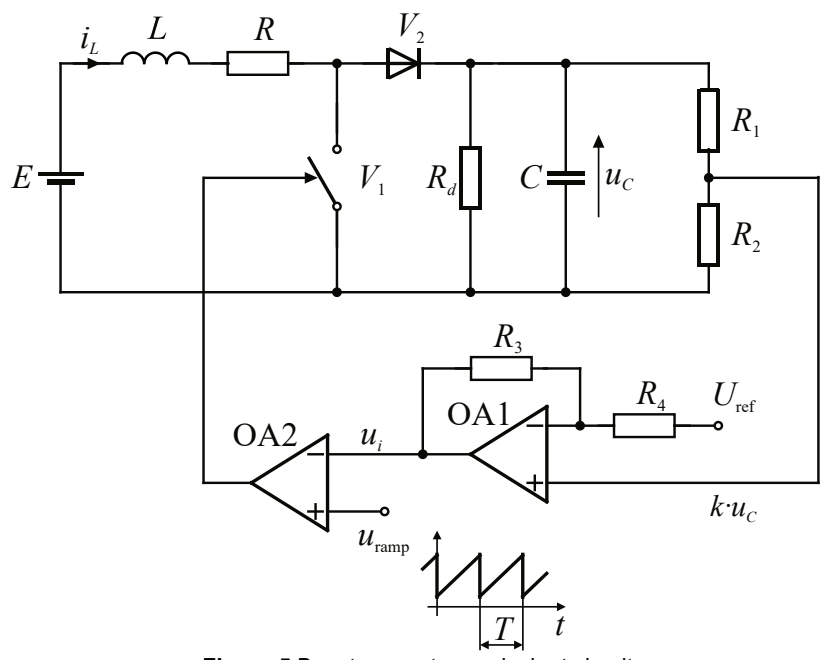

Figure 5 Boost converter equivalent circuit

When $u_{\text {ramp }} \geq u_{i}$, the controllable switch $V_{1}$ is in the $\mathrm{ON}$-state and the diode $V_{2}$ is in the OFF-state. The state equations are:

$$
\frac{\mathrm{d} u_{C}}{\mathrm{~d} t}=\frac{1}{C_{d} R_{d}} u_{C} ; \quad \frac{\mathrm{d} i_{L}}{\mathrm{~d} t}=\frac{1}{L}\left(E-R i_{L}\right)
$$

where the capacitor voltage $u_{C}$ and inductor current $i_{L}$ are state variables.

When $u_{\text {ramp }}<u_{i}, i_{L}>0$ the controllable switch $V_{1}$ is in the OFF-state and the diode $V_{2}$ is in the $\mathrm{ON}$-state. The state equations are:

$$
\frac{\mathrm{d} u_{C}}{\mathrm{~d} t}=\frac{1}{C_{d}}\left(i_{L}-\frac{u_{C}}{R_{d}}\right) ; \quad \frac{\mathrm{d} i_{L}}{\mathrm{~d} t}=\frac{1}{L}\left(E-R i_{L}-u_{C}\right)
$$

When $u_{\text {ramp }}<u_{i}, i_{L}=0$ the controllable switch $V_{1}$ is in the OFF-state and the diode $V_{2}$ is in the OFF-state. The state equations are:

$\frac{\mathrm{d} u_{C}}{\mathrm{~d} t}=\frac{1}{C_{d} R_{d}} u_{C} ; \quad i_{L}=0$

According to Fig. 5 the input voltage $u_{i}$ of the operational amplifier OA2 is given by:

$u_{i}=-\frac{R_{3}}{R_{4}} U_{\text {ref }}+\left(1+\frac{R_{3}}{R_{4}}\right) k u_{C}$

where $k$ is feedback coefficient defined as:

$k=\frac{R_{2}}{R_{1}+R_{2}}$

and is adjusted by changing the value of resistor $R_{1}$. The external sawtooth ramp voltage

$u_{\text {ramp }}=\frac{3}{T} t+0,7$

is obtained by measurements carried out on the pulse-width modulator MC34060A.

\subsection{Simulation Tool for Identification of Steady-States}

All simulations were done for a fixed set of parameters: $R_{1}=21,56 \mathrm{k} \Omega$, (adjusted to ensure output voltage in range from $27 \mathrm{~V}$ to $28 \mathrm{~V}) R_{2}=4,7 \mathrm{k} \Omega$, $R_{3}=33 \mathrm{k} \Omega, \quad R_{4}=3,9 \mathrm{k} \Omega, \quad R=0,7 \Omega, \quad L=1 \mathrm{mH}$, $C_{d}=100 \mu \mathrm{F}, R_{d}=39 \Omega, U_{\text {ref }}=5 \mathrm{~V}$ and $T=200 \mu \mathrm{s}$. Varied set of parameters i.e. bifurcations parameters were: the input voltage $E$, initial value of inductor current $i_{L}(0)$, initial value of capacitor voltage $u_{C}(0)$ and feedback coefficient $k$. The fourth-order Runge-Kutta method of numerical integration with the fixed step size of integration $h=20$ ns was used.

It is necessary to reproduce by simulation different type of steady-state responses of the boost converter, from higher periodic steady-state responses to chaos. It includes coexistence of two different steady-state solutions depending on initial conditions for the same value of input voltage. It is convenient to base identification of steadystate type on Poincaré method by which state variables are sampled by sawtooth voltage period, Fig. 6 . The number $N$ of different sampled values of state variable corresponds to the period- $N$ type of steady-state. The diagram in which these dots are represented as a function of circuit parameter is called one-parameter bifurcation diagram. The number $N$ of dots in one-parameter bifurcation diagram at a fixed value of circuit parameter corresponds to the period- $N$ type of steady-state. So, the information of steady-state type is contained in a line perpendicular to circuit parameter axis. More information about zone of steady-state type in a diagram is available by use of two-parameter bifurcation 
diagram. In that case both axes of diagram represent circuit parameter and the dot in diagram represents steady-state type. It is convenient to represent each steady-state type by different colour or by different texture. For this purpose the additional computer routine written in $\mathrm{C}+$ program code is implemented.

a)

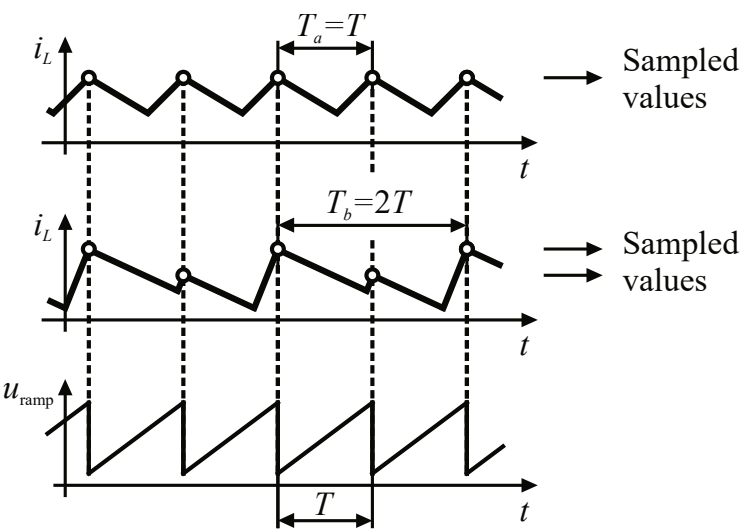

Figure 6 Sampling of inductor current. (a) All samples of period-1 steady-state have the same value; (b) All samples of period-2 steady-state have two different values. Sampled valued are marked with circles.

State variable waveforms and both one-parameter and two-parameter bifurcation diagrams were used as procedure for obtaining hysteretic bifurcation model of the chosen boost converter. All bifurcation diagrams are presented in time interval from about $100 \mathrm{~T}$ to $150 \mathrm{~T}$ to ensure the difference between two successive periods is small enough, i.e. to achieve the steady-state.

\subsubsection{Two-Parameter Bifurcation Diagrams}

As is known, coexisting attractors which caused hysteretic behaviour are a consequence of different initial conditions. The goal is to obtain different steady-state solutions at the same input voltage but with different initial conditions. In that manner steady-states in vicinity of point of hysteresis will be researched. It means that all simulation has to be carried on with input voltage in range $E \epsilon[22,9 \mathrm{~V}$, 23,2 V]. Besides, the converter's steady-states are sensitive to feedback coefficient value, so it is reasonable to investigate its impact on appearance of coexisting steadystate solutions.

The most information about steady-state type can be obtained from two-parameter bifurcation diagrams. Therefore, two-parameter bifurcation diagrams are computed. Still, there are several combinations of twoparameter bifurcation diagrams. To choose the combination of parameters which is the most convenient for further research it is necessary to set the range of parameters as small as possible to shorten the research. Here is the list of circuit parameters and initial conditions of state variables based on measurements and mathematical model of the converter used in simulations:

$E \in[22,9 \mathrm{~V}, 23,2 \mathrm{~V}]$,

$u_{C}(0) \in[26,7 \mathrm{~V}, 28,1 \mathrm{~V}]$,

$i_{L}(0) \in[0,2 \mathrm{~A}]$,

$k \in[0,16,0,18]$.

Using this range of parameters and trial and error method every of steady-state types in vicinity of point of hysteresis has to be obtained by simulation.
Two-parameter bifurcation diagrams of feedback coefficient with initial inductor current, with fixed initial capacitor voltage and varied input voltage are shown in Fig. 7. Areas of coexisting period-2 and period-3 steadystate solution are of interest.Three characteristic diagrams are shown. The area with constant feedback coefficient $k=0,168$ and with coexisting period-2 and period-3 steady-state solution is noticed. The diagrams are in correspodence with results obtained by the measurement shown in Fig. 4. When input voltage is less or about $E=23,05 \mathrm{~V}$ there is an area of coexistence of period-2 and period-3 steady-state solution and when input voltage is $E=23,15 \mathrm{~V}$ there is no area of coexistence of period-2 and period-3 steady-state solution. It means that corresponding value of input voltage is outside hysteretic region. When considering the input voltage as bifurcation parameter the point of hysteres is obtained by simulation in range $E \in[23,05 \mathrm{~V}, 23,15 \mathrm{~V}]$ while in measurement this point is at $23,02 \mathrm{~V}$ and $23,1 \mathrm{~V}$. The true value is not known, but all values mutually deviate less than $1 \%$ what is considered as acceptable. In vicinity of point of hysteresis even the small variations (of less than $1 \%$ ) of bifurcation parameter $E$ cause the change of the steady-state.

$$
u_{C}(0)=27,2 \mathrm{~V}
$$

a) $E=22,9 \mathrm{~V}$

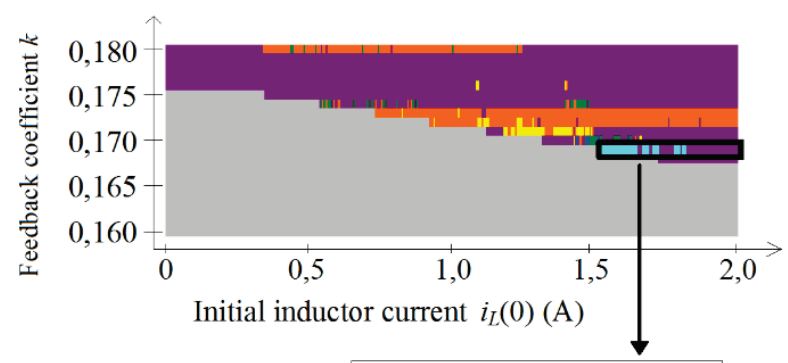

b) $E=23,05 \mathrm{~V} \quad$ Coexistence of period-2 and

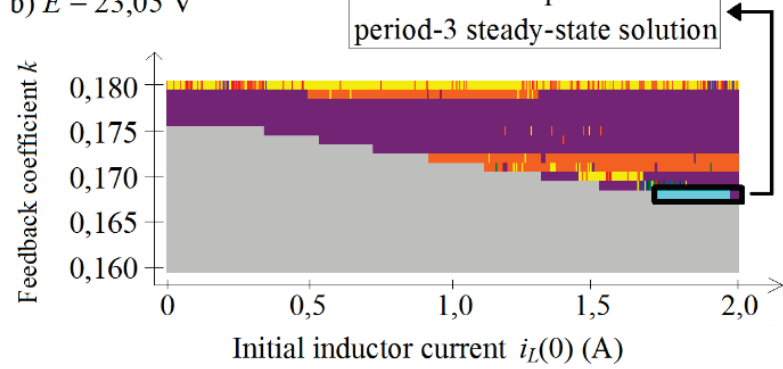

c) $E=23,15 \mathrm{~V}$

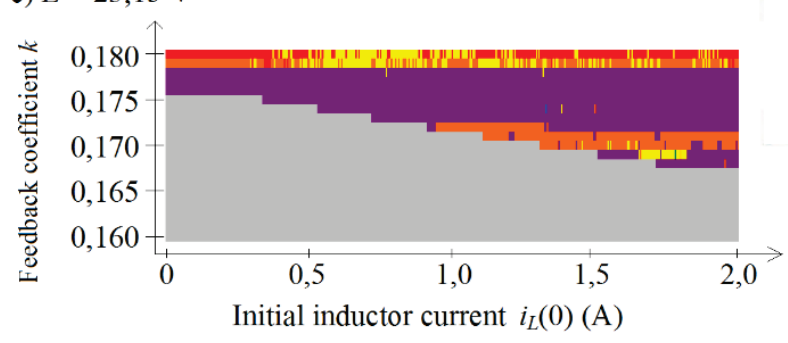

1234568 C The colours of steady-states

Figure 7 Two-parameter bifurcation diagrams of feedback coefficient with initial inductor current, with fixed initial capacitor voltage and varied input voltage. (a) $E=22,9 \mathrm{~V}$; (b) $E=23,05 \mathrm{~V}$; (c) $E=23,15 \mathrm{~V}$. Resolution is $\Delta i_{L}=0,005 \mathrm{~A}$, $\Delta k=0,001$.

With the change of initial capacitor voltage there is consequently a change in range of initial inductor current which causes coexistence of observed steady-state and vice 
versa. It is shown in Fig. 8. Two-parameter bifurcation diagram of feedback coefficient with initial inductor current, with input voltage fixed at $E=23,05 \mathrm{~V}$ and initial capacitor voltage $u_{C}(0)=28,15 \mathrm{~V}$ is shown in Fig. 8a. Two-parameter bifurcation diagram of feedback coefficient with initial capacitor voltage, with input voltage fixed at $E=23,05 \mathrm{~V}$ and initial inductor current $i_{L}(0)=1,92 \mathrm{~A}$ is shown in Fig. 8b. The area of coexistence of period-2 and period-3 steady-state solution in these two diagrams is the largest among all simulated diagrams with varied one of initial conditions with fixed another initial condition. Thus, considering the two diagrams with input voltage fixed at $E=23,05 \mathrm{~V}$ the range of initial conditions which cause coexistence of period- 2 and period- 3 steadystate solution is

$i_{L}(0) \in[0,6 \mathrm{~A}, 2 \mathrm{~A}]$

$u_{C}(0) \in[27,0 \mathrm{~V}, 28,15 \mathrm{~V}]$.

So, for a fixed input voltage the particular steady-state of coexisting steady-state solutions (period-2 or period-3) is determined by a pair of values $i_{L}(0)$ and $u_{C}(0)$.

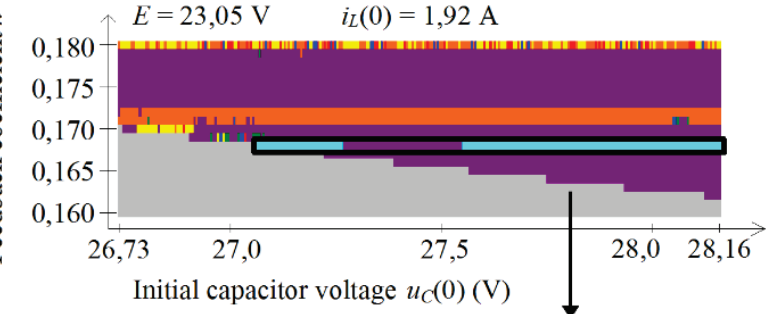

Coexistence of period-2 and

b)

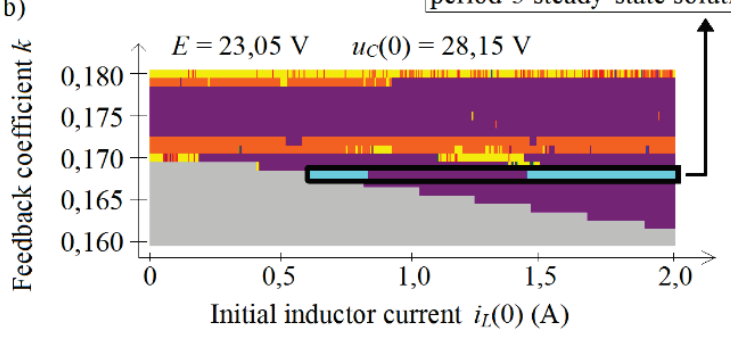

1254568 C The colours of steady-states

Figure 8 Two-parameter bifurcation diagram of feedback coefficient (a) with initial capacitor voltage at $E=23,05 \mathrm{~V}, i_{L}(0)=1,92 \mathrm{~A}$; (b) with initial inductor current at $E=23,05 \mathrm{~V}, u_{C}(0)=28,15 \mathrm{~V}$. Resolution is $\Delta u_{C}=0,005 \mathrm{~V}$, $\Delta i_{L}=0,005 \mathrm{~A}, \Delta k=0,001$.

Steady-states in hysteretic region are strongly sensitive on initial conditions. There is a variety of initial conditions which cause the particular steady-state. So, it is reasonably assumed that there is no unique model which accurately predicts all steady-states in hysteretic region regarding the tendency of change of input voltage.

\subsubsection{One-Parameter Bifurcation Diagrams}

It is well known that outside the hysteretic region steady-states do not depend on initial conditions. In that order, simulation and measurement of steady-state responses by change of input voltage was carried on. Types and ranges of simulated steady-state responses compared with measurements are shown in Tab. 1.
Table 1 Steady-state responses outside hysteretic region obtained by measurement and simulation.

\begin{tabular}{|c|c|c|}
\hline \multirow{2}{*}{$\begin{array}{c}\text { Steady-state } \\
\text { response }\end{array}$} & \multicolumn{2}{|c|}{ Input voltage $E(\mathrm{~V})$} \\
\hline & Measurement & Simulation \\
\hline Period-6 & $23,00-23,27$ & $23,03-23,22$ \\
\hline Period-9 & - & $23,22-23,32$ \\
\hline Chaos & $23,27-24,07$ & $23,32-24,06$ \\
\hline Period-4 & $24,07-24,42$ & $24,06-24,32$ \\
\hline Period-8 & $24,42-24,49$ & $24,32-24,50$ \\
\hline Chaos & $24,49-25,47$ & $24,50-25,46$ \\
\hline Period-5 & $25,47-25,68$ & $25,46-25,70$ \\
\hline Chaos & $25,68-26,63$ & $25,70-26,66$ \\
\hline Period-6 & $26,63-26,85$ & $26,66-26,76$ \\
\hline Chaos & $26,85-27$ & $26,76-27$ \\
\hline
\end{tabular}

Simulated steady-states of inductor currents correspond to the results obtained from the measurement. Only the period-9 steady-state response from $23,22 \mathrm{~V}$ to $23,32 \mathrm{~V}$ was not perceived by measuring. Its band was too narrow to be perceived regarding technical characteristics of the input DC source like input ripple and possibility of fine tuning. Proposed model was used to simulate oneparameter bifurcation diagram and state-variable waveforms outside the hysteretic region.

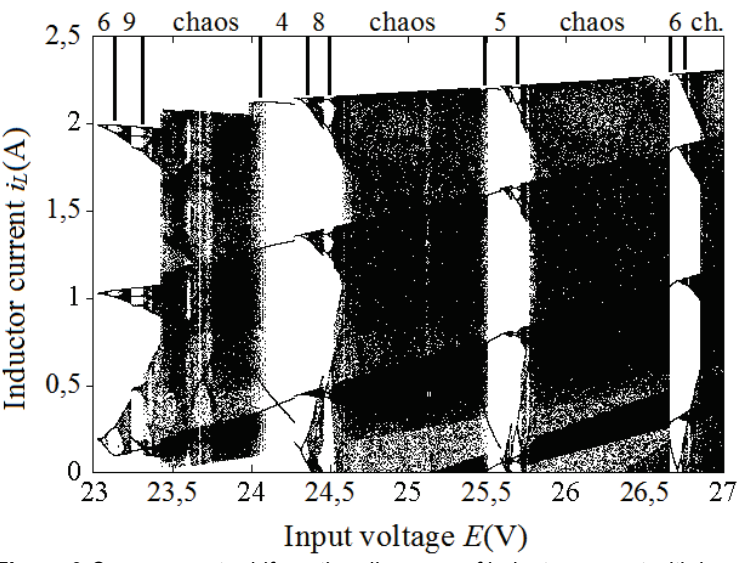

Figure 9 One-parameter bifurcation diagrams of inductor current with input voltage

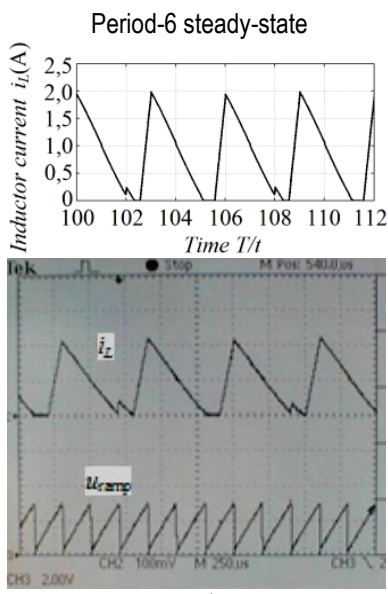

a)
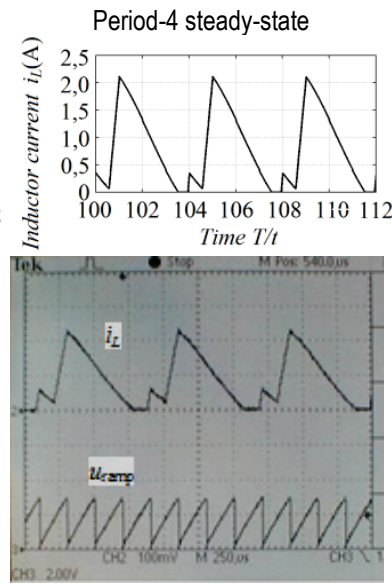

Figure 10 Inductor current waveforms obtained by simulation (upper) and by measurement (down). (a) $E=23,12 \mathrm{~V}$; (b) $E=24,08 \mathrm{~V}$. Sensitivity $1 \mathrm{~A} /$ div, $2 \mathrm{~V} / \mathrm{div}, 250 \mu \mathrm{s} / \mathrm{div}$.

One-parameter bifurcation diagram of inductor current with input voltage as a bifurcation parameter is shown in Fig. 9. Approximate regions of steady-states are indicated on the diagram. Furthermore, simulated inductor current waveforms of period- 6 and period- 4 steady-states are 
shown in Fig. 10 and compared with waveforms obtained by measurement. The bifurcation diagram and inductor waveforms agree very well to the measurement results. Proposed mathematical model is valid outside hysteretic region.

\section{CONCLUSION}

In the boost converter, a hysteretic behaviour of steady-state responses regarding the tendency of change of input voltage is experimentally confirmed. Proposed mathematical model is validated outside hysteretic region. Using two-parameter bifurcation diagrams by proper choice of initial conditions coexistence of two steady-state solutions and consequently existence of hysteretic behaviour is confirmed inside the hysteretic region. There is a variety of combinations of initial conditions of the state variables causing hysteretic. In hysteretic region the existing steady-state is strongly sensitive on initial conditions. Initial conditions are not under the control during measurement. Nevertheless, the range of initial conditions for a particular initial inductor current and initial capacitor voltage causing hysteretic behaviour can be identified by using the proposed procedure. Future work will focus on physical realisation of electronic switch for control of initial conditions during measurements.

\section{REFERENCES}

[1] Singh, B., Singh, S., Chandra, A., \& Al-Haddad, K. (2011) Comprehensive study of single-phase AC-DC power factor corrected converters with high-frequency isolation. IEEE transactions on Industrial Informatics, 7(4), 540-556. https://doi.org/10.1109/TII.2011.2166798

[2] Al-Hindawi, M. M., Abusorrah, A., Al-Turki,Y., Giaouris, D., Mandal, K., \& Banerjee, S. (2014). Nonlinear dynamics and bifurcation analysis of a boost converter for battery charging in photovoltaic applications. International Journal of Bifurcation and Chaos, 24(11). https://doi.org/10.1142/S0218127414501429

[3] Chang, W. H., Chen, D., Nien, H. S., \& Chen, C. H. (2008) A digital boost converter to drive white LEDs. Proceedings IEEE Applied Power Electronics Conference, 558-564.

[4] Alexander, I. \& Cook. A. (2016). Step-up DC-DC converter for automotive application. Power Electronics and Applications (EPE'16 ECCE Europe).

[5] Tse, C. K. (2004). Complex behaviour of switching power converters. CRC Press, Boca Raton.

[6] Chan, W. C. Y. \& Tse, C. K. (1997). Study of Bifurcations in Current Programmed DC/DC Boost Converters: From Quasi Periodicity to Period Doubling. IEEE Transactions on circuits and systems-I, Fundamental theory and applications, 44(12), 1129-1142. https://doi.org/10.1109/81.645154

[7] Banerjee, S. \& Chakrabarty, K. (1998). Nonlinear modeling and bifurcations in the boost converter. IEEE Transactions on Power Electronics, 13(2), 252-260. https://doi.org/10.1109/63.662832

[8] Pelin, D., Flegar, I., \& Miličević, K. (2009). Identification of steady-state responses of the boost converter. Technical gazette, 16(2), 9-15.

[9] Pikulin, D. (2013). Subharmonic Oscillations and Chaos in DC-DC Switching Converters. Elektronika ir Elektrotechnika, 19(4), 33-36. https://doi.org/10.5755/j01.eee.19.4.4054
[10] Strogatz, S. (1994). Nonlinear dynamics and chaos, Perseus Books publishing, Reading.

[11] Alligood, K. T., Sauer, T. D., \& York, J. A. (1996). Chaos An introduction to dynamical systems, Springer-Verlag, New York.

[12] Gupta, A., Banerjee, S., \& Kastha, D. (2003). Experimental study of bifurcations in the current controlled dc-dc buckboost converter. National conference of on nonlinear systems \& dynamics / India, 333-336.

[13] Aroudi, A. E. \& Leyva, R. (2001). Quasi-Periodic Route to Chaos in a PWM Voltage-Controlled DC-DC Boost Converter. IEEE Transactions on circuits and systems-I, Fundamental theory and applications, 48(8), 967-978. https://doi.org/10.1109/81.940187

[14] Zhusubaliyev, Z. T., Soukhoterin, E. A., \& Mosekilde, E. (2003). Quasi-Periodicity and Border-Collision Bifurcations in a DC-DC Converter with Pulsewidth Modulation. IEEE Transactions on circuits and systems-I, Fundamental theory and applications, 50(8), 1047-1057. https://doi.org/10.1109/TCSI.2003.815196

[15] Aroudi, A. E., Moreno-Font, V., \& Benadero, L. (2009). Dynamical Analysis of an Interleaved Single Inductor TITO Switching Regulator. Mathematical Problems in Engineering, 1-19. https://doi.org/10.1155/2009/946245

[16] Zhusubaliyev, Z. T., Mosekilde, E., Maity, S., Mohanan, S., \& Banerjee, S. (2006). Border collision route to quasiperiodicity: Numerical investigation and experimental confirmation. Chaos 16, p 023122. https://doi.org/10.1063/1.2208565

[17] Kolokolov, Y. V. \& Monovskaya, A. V. (2015). Experimental Identification of Uncertainties in Dynamics of PWM Buck Converter. IFAC-PapersOnLine, 48(1), 366371. https://doi.org/10.1016/j.ffacol.2015.09.213

\section{Contact information:}

Denis PELIN, PhD, Associate Professor Josip Juraj Strossmayer University of Osijek

Faculty of Electrical Engineering, Computer Science and Information Technology Osijek

Kneza Trpimira 2b 31000 Osijek, Croatia

dpelin@ferit.hr

Željko STOJANOVIĆ, Senior lecturer

Zagreb University of Applied Sciences Department of Electrical Engineering Konavoska 2, 10000 Zagreb, Croatia zeljko.stojanovic@tvz.hr 